\title{
Editorial: Becoming a Referee
}

Journal referees or peer reviewers are responsible academics or practitioners who aim to contribute to the development of research in their area of expertise. They are willing to evaluate the quality of work undertaken by other researchers highlighting the strengths and weaknesses of the work, and ensuring the accuracy and rigor of the research before widely disseminating it through a journal [1].

Referees from our journal are from diverse backgrounds: Computer Science, Information Systems, Business, Law, and the Social Science disciplines among others. This makes it difficult to apply exactly the same standards for the review of articles and also makes it difficult to provide very terse guidelines for referees. The refereeing task is a difficult one, but it is also an informative process. A referee is able to contrast poor articles with good ones, at the same time learning about what to do (or not to do) when the referee is writing his/her own paper. Volunteering time to review papers keeps one abreast of the latest developments in their area of research as well as providing exposure to cutting edge research methods. The referee's role is very important for the research community at large, and also can provide a high degree of prestige within the broader research network. Providing well-prepared, thoughtful and critical reviews can earn a referee instantaneous reputability, at the same time a poor review can reflect negatively on the general skills of the referee. Editors have great appreciation for referees that achieve excellence in their aim of providing well justified evaluations and quality feedback to authors. Most journals provide referee's certificates to highlight the tasks undertaken by the academic or practitioner. Achieving excellence as a referee over several years may also be the key to a future role on the Editorial Board of a journal. Perhaps discouraging is that the referee's task is often not acknowledged or rewarded by their academic institution. This important role in the advancement of the research community and the success of university departments has little bearing when the academic who is a referee is applying for promotion or during annual employment evaluations. We believe that university management should give more importance to the referee's task when setting procedures for evaluation and promotion of academics.

Becoming a referee may look simple, but becoming a good referee is difficult and sometimes misunderstood by those undertaking the task. The main role of the referee is to decide whether or not an article makes a sufficient contribution to the specific field of research [3]. Smith describes this contribution as: new and interesting research results; new and insightful synthesis of existing results; a useful survey of, or tutorial, on a field; or a combination of them [3]. A good referee's report should have four main components [3]:

- Summarize the article in a few sentences to ensure that you understand the article, and for the use of the editor

- Evaluate the validity and significance of the research objectives

- Evaluate the quality of the actual work presented in the article (methodology and rigor, structure, techniques, models, validation methods, results, discussion)

- Provide an overall recommendation to accept or reject the article.

Whatever recommendation is deemed appropriate by the referee, it must be clearly justified, especially when it is a rejection because the author will want to receive feedback on the main reasons the article was rejected. It is always necessary to distinguish between those research articles that may have a chance for publication after substantial changes have been made (in the same or a different journal), and those that do not. An acceptance will require the referee to list the changes that are necessary and those that are suggested [3]. We recommend reading Smith for a detailed understanding of how to evaluate a research article and make recommendations [3].

While it is true that the technical evaluation of a research article is important, the respect granted to authors through the refereeing process is also very important. Harrison reminds us that all 
referees are also authors, but we normally have different feelings depending on whether we are wearing the hat of an author or a referee. The former makes us feel creative, while the latter make us take a more destructive role [2].

Harrison [2] provides a bill of rights for authors. We have listed this below with additional comments, where appropriate, from a list of referee's responsibilities from the council of science editors [1]:

- Manuscript authors have a right to respectful and courteous interpersonal treatment. Always[2].

Those referees who agree to assess the work of another researcher and comment upon its importance, accuracy, clarity, and its usefulness to the research community and the world, have an important responsibility. It is their obligation to treat the author and the manuscript with respect [1].

- Manuscript authors have a right to a full and careful reading of their manuscripts [2].

When referees do not have the appropriate time to carefully read and assess the manuscript they have been asked to review, they must decline to referee it.

- Manuscript authors have a right to expect criticisms of their work to follow the same standards of logic and evidence applied to themselves [2].

Referees must provide an honest and clear assessment of the value of the research. An appropriate assessment includes an analysis of the strengths and weaknesses of the research presented, suggestions for improving the study making it more complete or relevant, specific questions for the authors to address with the aim of making their study acceptable and useful to the audience for which the results presented in the research article are intended [1].

- Manuscript authors have a right to expect criticisms of their work will be prioritized [2].

Referees should clearly indicate which criticisms and related suggestions are more important than others. If the referee believes that the manuscript suffers from a major flaw, this should be indicated early in the referee report.

- Manuscript authors have a right to get feedback about their work in a reasonable span of time [2].

Referees must fulfill their responsibility simultaneously to the research community and to the journal that has requested their assistance. By rigorously assessing a given research manuscript within the time limits specified by the journal, they meet their responsibility to the research community in full [1].

The responsibilities of peer reviewers or referees, as presented in the editorial policy statements by the council of science editors [1], include other important issues. Referees must inform the editors or decline to assess a manuscript when they:

- have a bias against either the manuscript authors or the research presented in it

- have a conflict of interest with the research presented in the manuscript or the sponsors of the research

- are not truly knowledgeable about the area of research in the manuscript they have been asked to assess.

Referees must also maintain confidentiality about the manuscripts they assess. The use of the data from such manuscripts before the work is published is inappropriate. It is equally inappropriate to share the data with colleagues, or to reproduce the manuscript for any purpose [1].

Referees must not use the refereeing process as a means to further their own research objectives, specifically by asking authors to respond to questions that are of interest to the referee but are not questions the original study was designed to answer [1].

Referees should not use their assessments as an opportunity to suggest to authors that their own published research be cited [1]. 
At the Journal of Theoretical and Applied Electronic Commerce Research we are aware of the high degree of difficulty implied by the nature of the referee's task, and also that the task is not rewarded at most institutions, therefore, we will reward referees not only with a referee's certificate, but we will also give a Referee of the Year Award. We will look for frequent reviewers who have provided outstanding review reports according to the Editor-in-Chief and Co-Editors.

We would like to encourage those that have not participated as referees in the past because of time constraints or other factors, to consider becoming referees, and give their time and expertise to the research community of which they are important members. To become a referee for the Journal of Theoretical and Applied Electronic Commerce Research, please email the Editor-inChief (jtaer@utalca.cl) offering your expertise in specific areas of e-Commerce.

\section{Narciso Cerpa \\ Editor-in-Chief}

jtaer@utalca.cl

April 2007

\section{References}

[1] Editorial Policy Statements Approved by the CSE Board of Directors (2007). Responsibilities and Rights of Peer Reviewers. Council of Science Editors. [Online]. Available: http://www.councilscienceeditors.org/services/draft_approved.cfm\#Paragraphsix.

[2] Harrison, D., A., 2002. Obligations and Obfuscations in the Review Process, Academy of Management Journal, Vol. 45, No. 6. pp. 1079-1084.

[3] Smith, A. J., 1990, The Task of the Referee, Computer, April, pp. 65-71. 